\title{
Sirt6 suppresses hepatocellular carcinoma cell growth via inhibiting the extracellular signal-regulated kinase signaling pathway
}

\author{
ZHI-GAO ZHANG and CHENG-YONG QIN
}

\author{
Department of Gastroenterology, Provincial Hospital Affiliated to Shandong University, Jinan, Shandong 250021, P.R. China
}

Received May 2, 2013; Accepted December 17, 2013

DOI: $10.3892 / \mathrm{mmr} .2013 .1879$

\begin{abstract}
Sirt6, a member of the mammalian sirtuin family, is a protein that is located in the nucleus and is an $\mathrm{NAD}^{+}$-dependent deacetylase important in the control of metabolic activity and genome stability. Recently, several studies have demonstrated the potential role of Sirt6 in tumor biology; however, the role of Sirt6 in hepatocellular carcinoma (HCC) remains unclear. In the present study, Sirt6 protein expression was found to be downregulated in human HCC tissue compared with adjacent normal tissue. Knockdown of Sirt6 promoted growth of the HepG2 HCC cell line, whereas overexpression of Sirt6 inhibited the growth of HepG2 cells. Overexpression of Sirt6 induced apoptosis in HepG2 cells, which was demonstrated by a terminal deoxynucleotidyl-transferase-mediated dUTP nick end labeling assay and cleaved caspase-3 immunoblotting. Furthermore, overexpression of Sirt6 decreased intracellular reactive oxygen species and superoxide anion levels. Finally, overexpression of Sirt6 inhibited phosphorylation of extracellular signal-regulated kinases $1 / 2$ (ERK1/2), and blocking the ERK1/2 pathway with chemical-specific inhibitor U0126, attenuated the tumor suppressive effect of overexpression of Sirt6. Collectively, these data suggest that Sirt6 is a tumor suppressor in HCC cells and may be a promising therapeutic target in $\mathrm{HCC}$.
\end{abstract}

\section{Introduction}

Hepatocellular carcinoma (HCC) is a challenging malignancy of global importance associated with high prevalence and mortality (1). Previously, HCC was predominant in undeveloped or less-developed countries; however, incidence of HCC has increased recently in developed regions, including Western

Correspondence to: Professor Cheng-Yong Qin, Department of Gastroenterology, Provincial Hospital Affiliated to Shandong University, 324 Jingwu Weiqi Road, Jinan, Shandong 250021, P.R. China

E-mail: cyqin01@126.com

Key words: Sirt6, hepatocellular carcinoma, apoptosis, reactive oxygen species, extracellular signal-regulated kinase 1/2
Europe, the United States and Japan (2-3). HCC has several noteworthy epidemiological features, including oncogene mutation, ethnic groups and the presence of several well-documented environmental potentially preventable risk factors, such as hepatitis $C$ virus infection (4,5). Although there has been a growing understanding of the molecular mechanisms underlying hepatocarcinogenesis in recent years (1), the causes and biology of HCC are not yet fully understood. Moreover, the efficacies of current treatment for HCC are unsatisfactory (6) resulting in poor prognosis with a median survival time of four months (2).

Sirtuins, mammalian homologs of the yeast protein silent information regulator 2 (Sir2), are a unique subclass of deacetylases and mono-ADP-ribosyltransferases that use NAD ${ }^{+}$ as a cosubstrate (7). There are seven members of the sirtuin family in mammals (Sirt1-Sirt7) (7) These proteins influence a wide array of pathophysiological processes, including DNA repair, cell survival, stress responses, metabolic homeostasis and aging (7). Sirt1, the most studied member of the family, has been implicated in carcinogenesis and cancer progression, and is considered to be a therapeutic target (8). Sirtl induces histone deacetylation and methylation, promoter $\mathrm{CpG}$ island methylation, transcriptional repression and deacetylation of tumor suppressor proteins (8). Sirt1 was reported to have a bidirectional effect on tumor initiation, progression and drug resistance; it can operate as a tumor suppressor or as an oncogenic factor, depending on the context and the study conditions (8). Sirt3 is another member of the sirtuin family, which preferentially localizes to mitochondria and is involved in mitochondrial energy production and substrate oxidation (9-10). Unlike Sirt1, accumulating evidence suggests that Sirt3 is a tumor suppressor in breast cancer (11), oral cancer (12) and HCC (13).

Sirt6 is a less-studied nuclear sirtuin member. Thus far, Sirt6 has been found to regulate glucose homeostasis in the liver (14) and maintain genome stability (15-16). In 2012, Min et al (17) showed that Sirt6-dependent inhibition of survivin contributed to activator protein-1 binding site-induced tumor suppression. Another group confirmed that deletion of Sirt6 in vivo increased the number, size and aggressiveness of tumors (18). However, evidence from other studies indicates that Sirt6 may be tumorigenic in pancreatic cancer (19) and breast cancer (20). Consequently, the exact role of Sirt6 in cancer is still being analyzed. In the present study, 
the expression of Sirt6 in human HCC tissues and the potential role of Sirt6 in HCC were investigated.

\section{Materials and Methods}

Reagents. Antibodies against Sirt6 and $\beta$-actin were purchased from Sigma (St. Louis, MO, USA). Antibodies against cleaved caspase-3, phosphorylated extracellular signal-regulated kinase (ERK1/2), total-ERK1/2 and U0126 were purchased from Cell Signaling Technology, Inc. (Danvers, MA, USA). An immunofluorescence terminal deoxynucleotidyl transferase-mediated dUTP nick end labeling (TUNEL) kit was purchased from Promega Corporation (Madison, WI, USA). DAPI and dichlorofluorescein (DCF) were purchased from Invitrogen Life Technologies (Carslbad, CA, USA). Enhanced chemiluminescence and protease/phosphatase inhibitors were purchased from Pierce (Rockford, IL, USA).

Human HCC tissue. Four pairs of HCC and matched normal adjacent tissue extracts were obtained from Chinese patients who underwent surgical resection for diagnosis and therapy in the Provincial Hospital Affiliated to Shandong University (Jinan, China). Samples were obtained after receiving informed consent according to an established protocol approved by the Ethics Committee of Shandong University (Jinan, China).

Cell culture. HepG2 human HCC cells and HEK293 cells were obtained from the American Type Culture Collection (Manassas, VA, USA). Cells were cultured in Dulbecco's modified Eagle's medium supplemented with $10 \%$ (v/v) fetal bovine serum (FBS) in $95 \% \mathrm{O}_{2}$ and $5 \% \mathrm{CO}_{2}(21)$.

Plasmid construction. The adenovirus expressing Sirt6 (Ad-Sirt6) and the control adenovirus expressing green fluorescent protein (Ad-GFP) were generated using the Adenoviral Expression system (Cell Biolabs, Inc., San Diego, CA, USA) according to the manufacturer's instructions as described previously (22). Mouse Sirt6 mRNA was extracted from mouse liver tissue using TRIzol (Invitrogen Life Technologies) and was reverse transcribed with AMV to obtain cDNA. The full length of the mouse Sirt6 cDNA was amplified using polymerase chain reaction (PCR) with specific primers. Full length PCR products of Sir6 were then subcloned into the pacAd5 CMV-IRES vector (Cell Biolabs, Inc.). Next, pacAd5 CMV-IRES-Sirt6 and pacAd5 backbone vectors were linearized by PacI (New England Biolabs, Ipswich, MA, USA).

The short hairpin-RNA (shRNA)-induced RNA interference (RNAi) was achieved using the RAPAd ${ }^{\circledR}$ shRNA Adenoviral Expression system (Cell Biolabs, Inc.) according to the manufacturer's instructions. The nucleotide sequence for shRNA was designed using BLOCK-iT ${ }^{\mathrm{Tм}}$ RNAi Online Designer tool (Invitrogen Life Technologies). The following nucleotide sequence against human Sirt6 was used in this study: 5'-GGTCTGGCAGTCTTCCAGTGT-3'.

Generation of adenovirus. HEK293 cells were used to produce adenovirus. The purified linearized DNAs or plasmids were transfected into HEK293 cells using Lipofectamine 2000 reagent (Invitrogen Life Technologies). At $6 \mathrm{~h}$ after transfec- tion, the medium containing Lipofectamine 2000 was removed and novel medium was added. Adenovirus-containing HEK 293 cells and media were harvested on the 6th day post-transfection. Viruses were released by three freeze/thaw cycles and stored at $-80^{\circ} \mathrm{C}$. For virus transfection, $30 \mu \mathrm{l}$ viral stock solution was added into culture medium ( $2 \mathrm{ml})$ of HepG2 cells for $6 \mathrm{~h}$.

Cell proliferation assay. Cell counting kit-8 (CCK-8; Dojindo Laboratories, Kumamoto, Japan) was used to detect cell proliferation as described previously $(13,23)$. Ad-GFP and Ad-Sirt6-transfected cells $\left(3 \times 10^{4}\right)$ were seeded into 48 -well plates and cultured overnight to allow attachment. On the second day, cells were serum-starved for $8 \mathrm{~h}$, and FBS was added into medium. At 12, 24, 36 and 48 h, cells were incubated with $10 \mu \mathrm{l} \mathrm{CCK}-8$ solution for $2 \mathrm{~h}$, and the optical density at $450 \mathrm{~nm}$ was analyzed (Tecan Ultra 384 reader; Tecan, Männedorf, Switzerland). Experiments were performed in duplicate.

TUNEL assay. TUNEL staining was performed as described previously $(13,24)$. Cells were transfected with Ad-GFP or Ad-Sirt6 for $6 \mathrm{~h}$. At $24 \mathrm{~h}$ after treatment, cells were incubated in TUNEL reaction buffer in a humidified chamber for $1 \mathrm{~h}$ at $37^{\circ} \mathrm{C}$ in the dark, then rinsed four times with phosphate-buffered saline (PBS) and incubated with DAPI $(1 \mathrm{mg} / \mathrm{ml})$ for $15 \mathrm{~min}$. The stained cells were visualized using a fluorescence microscope (IX-71, Olympus, Tokyo, Japan). TUNEL-positive cells (green) were counted as apoptotic cells. Images were acquired digitally from a randomly selected pool of 10-15 fields under each condition (25).

Quantitative PCR analysis. qPCR analysis was performed on an OpticonDNA engine (MJ Research, Inc., St. Bruno, QC, Canada) using PrimerScript ${ }^{\circledR}$ RT Reagent kit (Takara Bio, Inc., Shiga, Japan) and normalized as described previously $(23,26)$. The total RNA was extracted form human tissue using TRIzol (Invitrogen Life Technologies) and $10 \mu \mathrm{g}$ RNA was used for first strand cDNA synthesis. cDNA (100 ng) was amplified using primers as follows: Sense: 5'-CGCAGTACGTCCGAGACACAGT-3' and antisense: 5'-TTGGTAGCCAGCGGCAGGTT-3' for Sirt6; and sense: 5'-GCACTCTTCCAGCCTTCCTTCC-3' and antisense: 5'-CCGCCAGACAGCACTGTGTT-3' for $\beta$-actin. The mRNA level of housekeeping gene $\beta$-actin served as a control.

Immunoblotting. Immunoblotting analyses of cell-extracts were performed as described previously (27-28). Human tissues or cells were lysed in $50 \mathrm{mM}$ Tris- $\mathrm{HCl}(\mathrm{pH} 7.5)$ and $1 \%$ SDS with protease/phosphatase inhibitor cocktail (Pierce), and then heated at $95^{\circ} \mathrm{C}$ for $10 \mathrm{~min}$. Samples were subjected to $10 \%$ SDS-PAGE, and transferred onto polyvinylidene fluoride membranes (Millipore, Milford, MA, USA) at 100V for 90 min. Following blocking in 5\% skimmed milk and PBS containing $0.1 \%$ Tween-20, membranes were incubated with primary antibodies (cleaved caspase-3 and Sirt6) followed by horseradish peroxidase-labeled secondary antibodies (Santa Cruz Biotechnology, Inc., Santa Cruz, CA, USA). The membranes were then detected using an enhanced chemiluminescence kit (Pierce). 
A

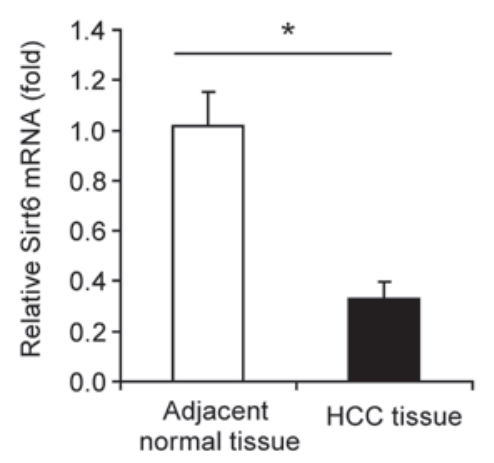

B

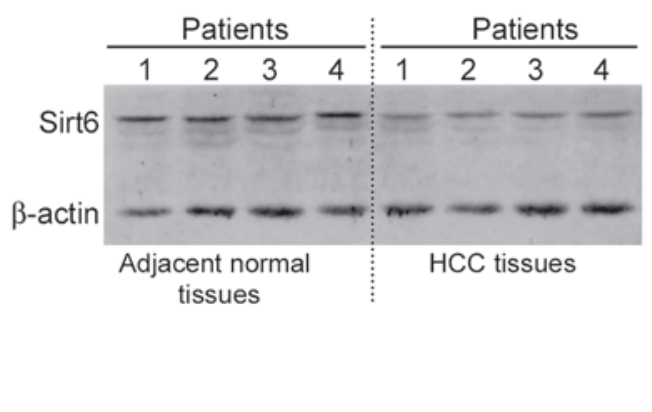

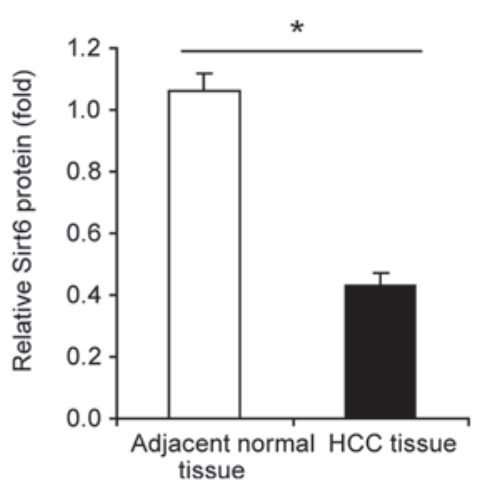

Figure 1. Downregulation of Sirt6 in human hepatocellular carcinoma (HCC) tissue. (A) The mRNA level of Sirt6 in human HCC tissue was measured by quantitative polymerase chain reaction analysis. (B) Immunoblotting and quantitative analysis of the expression of Sirt6 in human HCC tissue and normal adjacent tissue. ${ }^{*} \mathrm{P}<0.05$ versus normal tissue. Number of tissues $=4$.

A
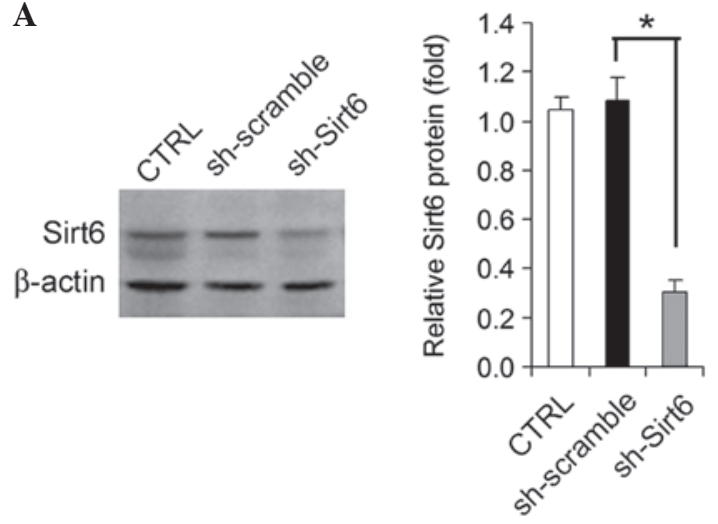

C

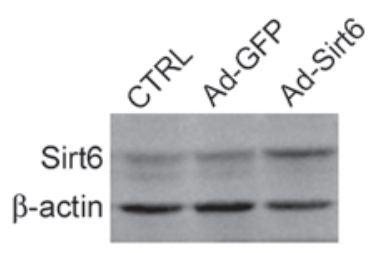

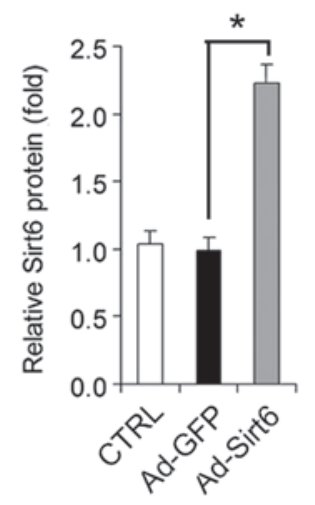

B

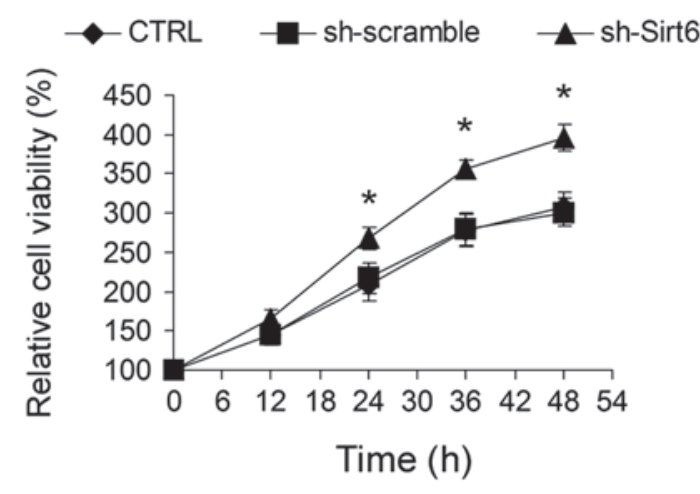

D

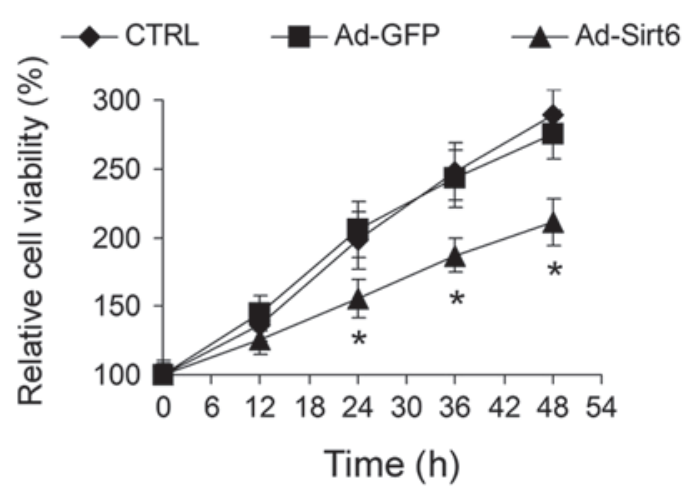

Figure 2. Modulation of Sirt6 affects hepatocellular carcinoma (HCC) cell growth. (A) Immunoblotting showing the short hairpin (sh)RNA-mediated knockdown of Sirt6 in HepG2 HCC cells and (B) cell growth curve of HepG2 HCC cells transfected with sh-scramble or sh-Sirt6. "P $<0.05$ versus sh-scramble (vector control). Number of tissues=8. (C) Immunoblotting showing the adenovirus-mediated overexpression of Sirt6 in HepG2 HCC cells and (D) cell growth curve of HepG2 HCC cells transfected with adenovirus expressing-green fluorescent protein (Ad-GFP) or Ad-Sirt6. " $\mathrm{P}<0.05$ versus Ad-GFP (vector control). Number of tissues $=8$.

ROS measurement. A DCF assay was used for the quantification of intracellular ROS as previously described (29). Ad-GFP and Ad-Sirt6 transfected cells were plated onto 96-well plates (5x $10^{4}$ cells/well) and loaded with $100 \mu \mathrm{M}$ DCF (Invitrogen Life Technologies) for $1 \mathrm{~h}$ at $37^{\circ} \mathrm{C}$. Cells were subsequently washed using PBS buffer. Fluorescence was measured using a fluorescence microplate reader (Tecan) with an excitation filter of $485 \mathrm{~nm}$ and an emission filter at $530 \mathrm{~nm}$.
Superoxide anion measurement. Ad-GFP- and Ad-Sirt6-transfected cells were seeded in 96-well plates and grown overnight. Following removal of the cell culture medium, the cells were washed with PBS, and $200 \mu 1$ of $25 \mu \mathrm{M}$ dihydroethidium (DHE) dissolved in PBS was added to each well for $1 \mathrm{~h}$. The fluorescence (DHE ex/em: 530/620 nm) was measured using a multiwell plate reader (Tecan) for $20 \mathrm{~min}$ at $37^{\circ} \mathrm{C}$. 
A
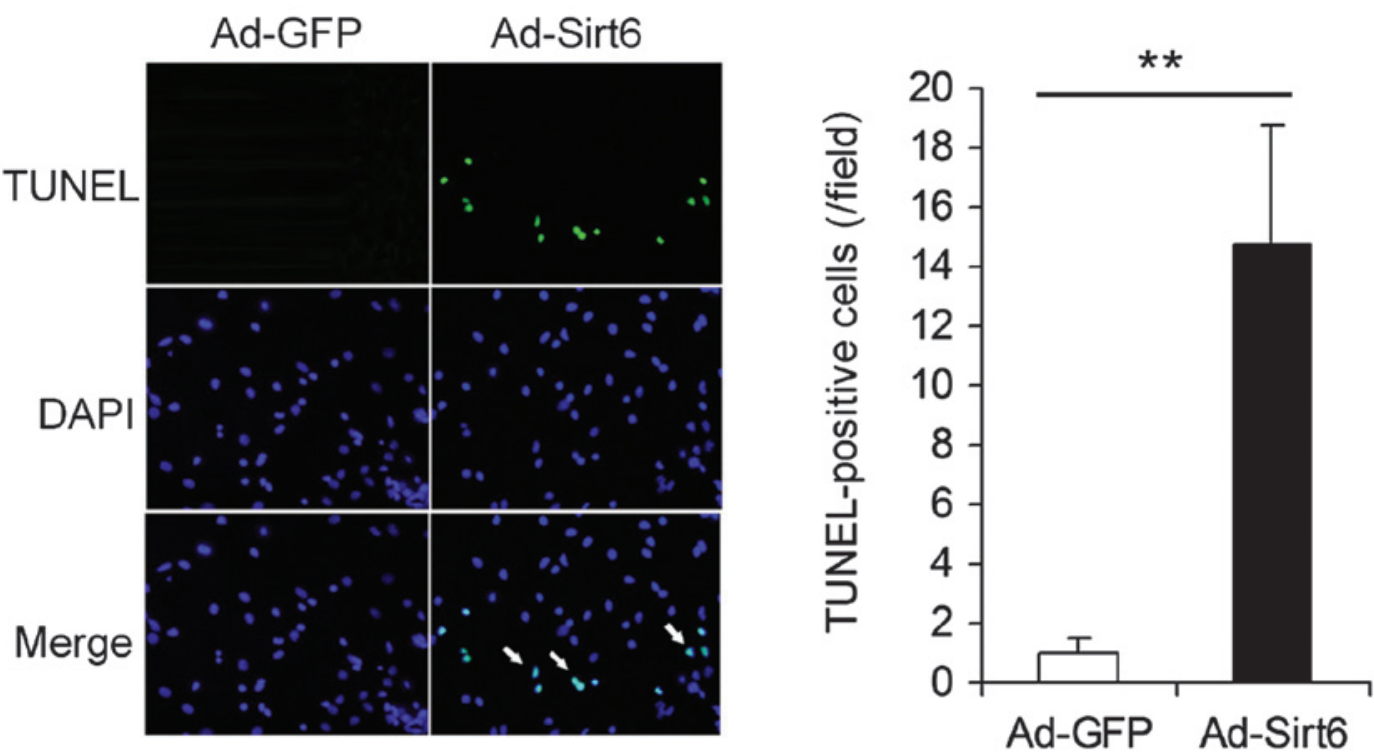

B
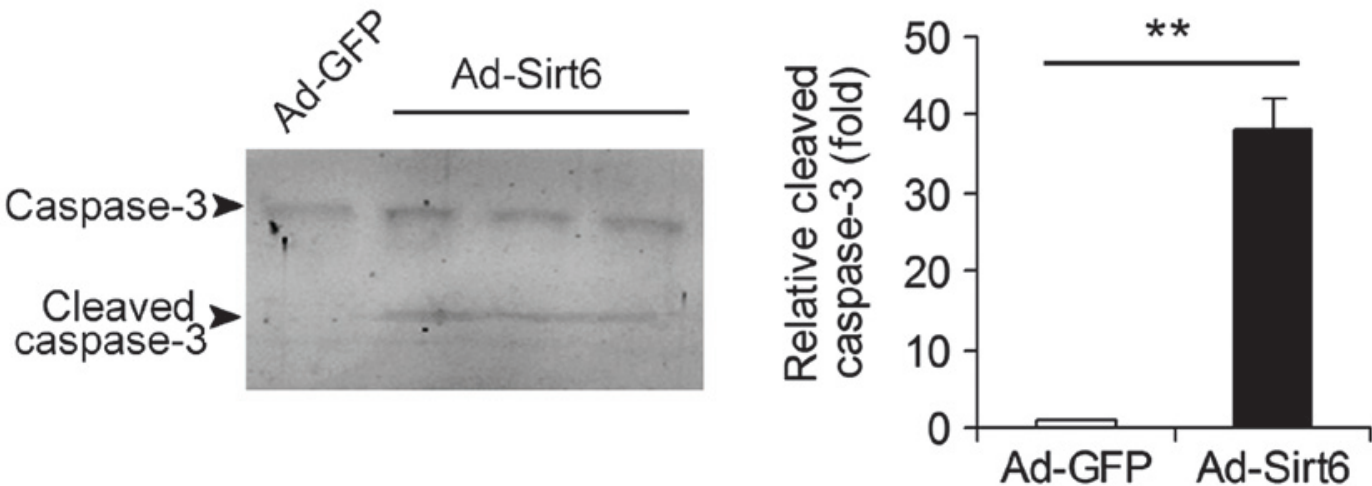

Figure 3. Apoptosis of hepatocellular carcinoma (HCC) cells induced by Sirt6 overexpression. (A) Terminal deoxynucleotidyl transferase-mediated dUTP nick end labeling (TUNEL) assay showed the apoptosis in HepG2 HCC cells overexpressing Sirt6. DAPI was used to stain the nucleus. Arrows indicate TUNELpositive cells. (B) Immunoblotting showing the cleaved caspase-3 in Sirt6 overexpressed HepG2 HCC cells. ${ }^{* *} \mathrm{P}<0.01$ versus Ad-GFP (control). Number of tissues $=8$.

Statistical analysis. Data are expressed as the mean \pm standard error of the mean. Differences were evaluated by two-tailed Student's t-test or analysis of variance followed by Tukey's post hoc test. $\mathrm{P}<0.05$ was considered to indicate a statistically significant difference.

\section{Results}

Downregulation of Sirt6 in human HCC tissue. Sirt6 mRNA levels were significantly downregulated $(35-40 \%, \mathrm{P}<0.05)$ in human HCC tissue compared with normal adjacent tissue (Fig. 1A). A marked downregulation of Sirt6 protein levels was also observed in human HCC tissue compared with normal adjacent tissue (Fig. 1B).

Sirt6 regulates Hep 62 cell growth. The effect of knockdown or overexpression of Sirt6 on HepG2 HCC cell growth in vitro was analyzed. Adenovirus-mediated knockdown of Sirt6 (Fig. 2A) by shRNA promoted HepG2 cell growth (Fig. 2B), whereas adenovirus-mediated overexpression of
Sirt6 (Fig. 2C) significantly inhibited HepG2 cell growth (Fig. 2D).

Overexpression of Sirt6 induces apoptosis in HepG2 HCC cells. The effects of overexpression of Sirt6 on the apoptosis of HCC cells were studied. Ad-GFP- and Ad-Sirt6-transfected cells were analyzed using the TUNEL assay. The apoptotic cells (TUNEL-positive cells, green) were detected in Ad-Sirt6 cells but not in control Ad-GFP cells (Fig. 3A). The protein levels of cleaved caspase-3, a key mediator and marker protein of apoptosis were also analyzed. Cleaved caspase- 3 expression was detected in cells overexpressing Sirt6 but not in control cells (Fig. 3B). These results indicate that overexpression of Sirt6 induces apoptosis in HepG2 HCC cells.

Overexpression of Sirt6 decreases oxidative stress in HCC cells. The influence of Sirt6 overexpression on oxidative stress in HepG2 HCC cells was investigated. A DCF assay showed that overexpression of Sirt6 significantly decreased the total ROS level in HepG2 cells (Fig. 4A). Moreover, overexpression 
A

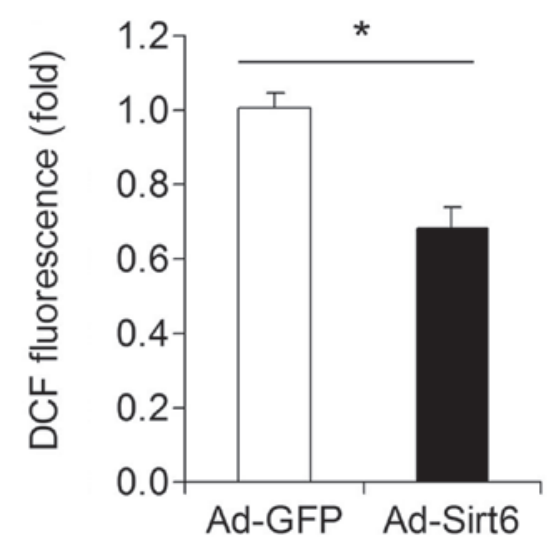

B

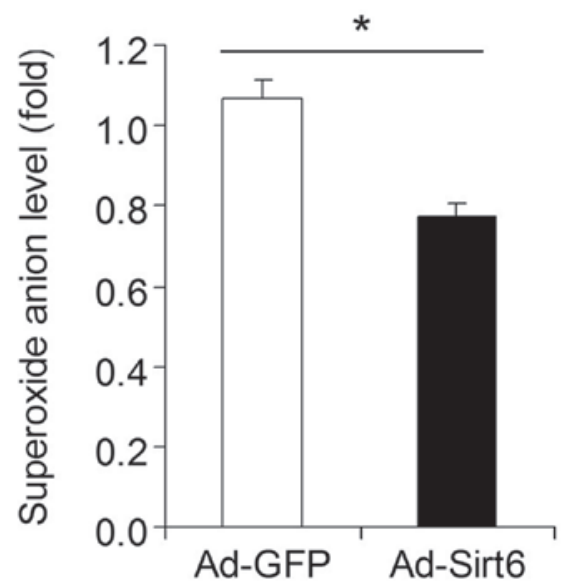

Figure 4. Sirt6 overexpression decreases reactive oxygen species (ROS) stress in hepatocellular carinoma (HCC) cells. (A) Dichlorofluorescein fluorescence assay showed that Sirt6 overexpression decreased ROS level in HCC cells. (B) Sirt6 overexpression decreased superoxide anion levels in HCC cells. ${ }^{*} \mathrm{P}<0.05$ versus adenovirus expressing green fluorescent protein (Ad-GFP). Number of tissues $=6$.

A

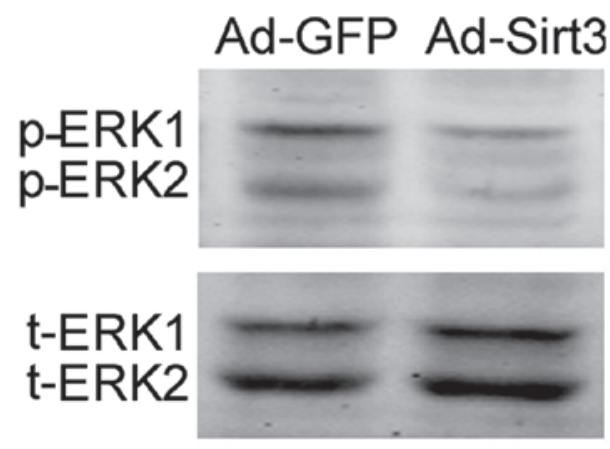

B

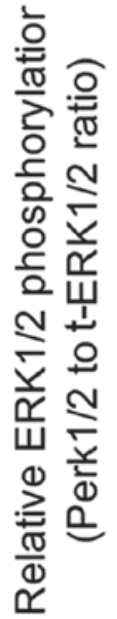

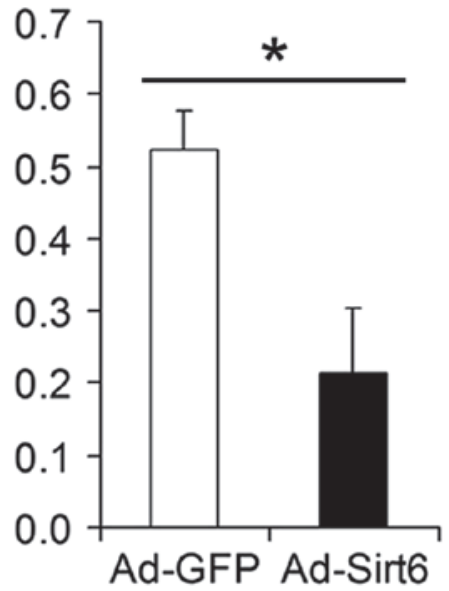

Figure 5. Influence of Sirt6 overexpression on activation of extracellular signal-regulated kinases 1/2 (ERK1/2) signaling pathway in hepatocellular carcinoma cells. Representative immunoblotting images and quantitative analysis of phosphorylation of ERK1/2 [ratio of phosphorylated ERK1/2 (p-ERK1/2) to total-ERK1/2 (t-ERK1/2)]. ${ }^{*} \mathrm{P}<0.05$ versus Ad-GFP. Number of tissues=6.

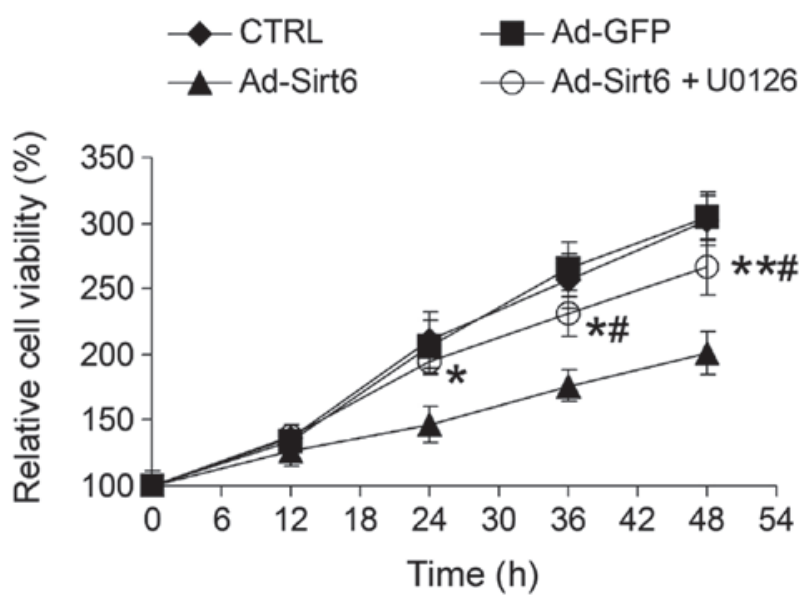

Figure 6. Sirt6 overexpression suppresses hepatocellular carcinoma (HCC) cell growth through regulating extracellular signal-regulated kinases 1/2 (ERK1/2) signaling pathway. Blocking the ERK1/2 signaling pathway with U0126 $(10 \mu \mathrm{m})$ attenuated the inhibitory effect of Sirt6 overexpression on HCC cell growth. ${ }^{*} \mathrm{P}<0.05$ versus Ad-Sirt6. Number of tissues $=6$. ${ }^{~} \mathrm{P}<0.05$ versus adenovirus expressing-green fluorescent protein (Ad-GFP). Number of tissues $=6$. of Sirt6 downregulated the superoxide anion level in HepG2 cells (Fig. 4B).

Overexpression of Sirt6 inhibits ERK1/2 phosphorylation in HCC cells. ERK1/2 is a major transducer of extracellular mitogenic signals that promote cell proliferation. The influence of the overexpression of Sirt6 on the phosphorylation of ERK1/2 was analyzed. Overexpression of Sirt6 significantly inhibited ERK1/2 phosphorylation in HCC cells (Fig. 5).

Sirt6 suppresses HCC cell growth via regulation of the ERK1/2 signaling pathway. To examine whether the altered ERK1/2 signaling pathway induced by Sirt6 overexpression was important for Sirt6-mediated tumor suppression, HepG2 cells were treated with U0126, a chemical inhibitor of the ERK1/2 pathway. U0126 markedly attenuated the inhibitory effect of Sirt6 on HCC cell growth (Fig. 6), suggesting that Sirt6 suppresses HCC cell growth via inhibition of the ERK1/2 signaling pathway. 


\section{Discussion}

In the present study, Sirt6 was markedly downregulated in HCC tissue compared with normal adjacent tissue. Using adenovirus-mediated knockdown and overexpression, modulation of Sirt6 affected the growth of HepG2 HCC cells. Further analyses, including a TUNEL assay and cleaved caspase-3 immunoblotting, revealed that Sirt6 overexpression promoted apoptosis in HepG2 HCC cells. In addition, Sirt6 overexpression decreased ROS/superoxide anion levels in HepG2 HCC cells. Finally, Sirt6 overexpression was found to inhibit phosphorylation of ERK1/2 in HepG2 HCC cells. Blocking the ERK1/2 pathway with U0126 attenuated the inhibitory effect of Sirt6 overexpression on HepG2 cell growth. These results suggest that Sirt6 is a tumor suppressor in HCC cells.

Several previous studies have demonstrated a change in Sirt6 expression in tumors. In a gene expression screening of endometrial carcinoma samples, Colas et al (30) revealed that Sirt6 was marginally upregulated. By contrast, in a recent study, Sebastián et al (18) reported that the Sirt6 locus was deleted in $35 \%$ of $~ 1,000$ cancer cell lines and in $62.5 \%$ and $29 \%$ of pancreatic and colorectal cancer cell lines respectively. Moreover, this group demonstrated that Sirt6 expression was downregulated in 36 pancreatic ductal adenocarcinomas and in 55 colorectal carcinomas. In head and neck squamous cell carcinomas, Lai et al (31) confirmed that Sirt6 was downregulated in cancerous tissues when compared with noncancerous tissues. To the best of our knowledge, the present study provides the first evidence of Sirt6 downregulation in human HCC tissue. A report showed impaired Sirt6 expression in c-Jun-deficient livers during tumor initiation in mice and Sirt6 expression in human HCC tissue (17); however, whether Sirt6 expression was different in human HCC tissues compared to normal tissues was not investigated in this study.

In the present study, shRNA-mediated knockdown of Sirt6 was identified to promote HepG2 cell growth, which was consistent with a previous report (18). The tumor suppressive effect of Sirt6 has also been demonstrated in cervical carcinoma, fibrosarcoma, primary breast tumor and metastatic breast tumor cell lines (32). In concordance with these results, the results of the present study provide evidence for the tumor suppressive effect of Sirt6 in HCC cells. Notably, Sirt6 overexpression appeared to induce apoptosis in a variety of cancer cell lines but not in normal, non-transformed cells (32). Additionally, mono-ADP-ribosyltransferase, but not deacetylase activity, was required for the tumor suppressive effect of Sirt6 (32).

As a member of mitogen-activated protein kinases, ERK1/2 mediates intracellular signaling pathways involved in proliferative functions, including meiosis, mitosis and postmitotic differentiation in cells (33). A number of different stimuli, including cytokines, growth factors, transforming agents and carcinogens, activate the ERK1/2 pathway to promote cell proliferation (33). Activated ERK1/2 is an important feature in $\mathrm{HCC}$ and multiple anticancer agents inhibit HCC cell growth via inhibition of ERK1/2 signaling (34). Previous studies revealed that Sirt1 activated the ERK1/2 pathway (35-36), whereas Sirt3 repressed the ERK1/2 signaling pathway (13). To the best of our knowledge, there has been no report on the association between ERK1/2 and Sirt6. Using immunoblotting, Sirt6 overexpression was found to inhibit the phosphorylation of ERK1/2 in HCC cells. In addition, blocking the ERK1/2 signaling pathway with the specific chemical inhibitor U0126, markedly attenuated the tumor suppressive effect of Sirt6. As ERK1/2 localizes to the cytoplasm and is translocated to the nucleus following phosphorylation (33), it is not known how Sirt6, a nuclear protein, regulates ERK1/2 phosphorylation. This is an important question that needs to be addressed in future studies.

In conclusion, the present study demonstrated that the expression of Sirt6 was decreased in human HCC tissue. Overexpression of Sirt6 in the HepG2 HCC cell line exhibited antitumor effects through the induction of apoptosis and the inhibition of the ERK1/2 signaling pathway. These findings on the regulation of HCC cell growth by Sirt6 may provide an improved understanding of HCC and aid in the possible development of therapeutic interventions.

\section{References}

1. El-Serag HB and Rudolph KL: Hepatocellular carcinoma: epidemiology and molecular carcinogenesis. Gastroenterology 132: 2557-2576, 2007

2. Jemal A, Bray F, Center MM, Ferlay J, Ward E and Forman D: Global cancer statistics. CA Cancer J Clin 61: 69-90, 2011.

3. Bosch FX, Ribes J, Díaz M and Cléries R: Primary liver cancer: worldwide incidence and trends. Gastroenterology 127 (5 Suppl 1): S5-S16, 2004.

4. Moradpour D, Penin F and Rice CM: Replication of hepatitis C virus. Nat Rev Microbiol 5: 453-463, 2007.

5. Yang JD and Roberts LR: Hepatocellular carcinoma: A global view. Nat Rev Gastroenterol Hepatol 7, 448-458, 2010.

6. Kozyreva ON, Chi D, Clark JW, et al: A multicenter retrospective study on clinical characteristics, treatment patterns, and outcome in elderly patients with hepatocellular carcinoma. Oncologist 16: 310-318, 2011.

7. Haigis MC and Guarente LP: Mammalian sirtuins - emerging roles in physiology, aging, and calorie restriction. Genes Dev 20: 2913-2921, 2006.

8. Liu T, Liu PY and Marshall GM: The critical role of the class III histone deacetylase SIRT1 in cancer. Cancer Res 69: 1702-1705, 2009.

9. Alhazzazi TY, Kamarajan P, Verdin E and Kapila YL: SIRT3 and cancer: tumor promoter or suppressor? Biochim Biophys Acta 1816: 80-88, 2011.

10. Hirschey MD, Shimazu T, Goetzman E, et al: SIRT3 regulates mitochondrial fatty-acid oxidation by reversible enzyme deacetylation. Nature 464: 121-125, 2010.

11. Finley LW, Carracedo A, Lee J, et al: SIRT3 opposes reprogramming of cancer cell metabolism through HIF1 $\alpha$ destabilization. Cancer Cell 19: 416-428, 2011.

12. Alhazzazi TY, Kamarajan P, Joo N, et al: Sirtuin-3 (SIRT3), a novel potential therapeutic target for oral cancer. Cancer 117: 1670-1678, 2011.

13. Zhang YY and Zhou LM: Sirt3 inhibits hepatocellular carcinoma cell growth through reducing Mdm2-mediated p53 degradation. Biochem Biophys Res Commun 423: 26-31, 2012.

14. Zhong L, D'Urso A, Toiber D, et al: The histone deacetylase Sirt6 regulates glucose homeostasis via Hiflalpha. Cell 140: 280-293, 2010.

15. Mao Z, Hine C, Tian X, et al: SIRT6 promotes DNA repair under stress by activating PARP1. Science 332: 1443-1446, 2011.

16. Kaidi A, Weinert BT, Choudhary C and Jackson SP: Human SIRT6 promotes DNA end resection through CtIP deacetylation. Science 329: 1348-1353, 2010.

17. Min L, Ji Y, Bakiri L, et al: Liver cancer initiation is controlled by AP-1 through SIRT6-dependent inhibition of survivin. Nat Cell Biol 14: 1203-1211, 2012.

18. Sebastián C, Zwaans BM, Silberman DM, et al: The histone deacetylase SIRT6 is a tumor suppressor that controls cancer metabolism. Cell 151: 1185-1199, 2012.

19. Bauer I, Grozio A, Lasigliè D, et al: The $\mathrm{NAD}^{+}$-dependent histone deacetylase SIRT6 promotes cytokine production and migration in pancreatic cancer cells by regulating $\mathrm{Ca}^{2+}$ responses. J Biol Chem 287: 40924-40937, 2012. 
20. Khongkow M, Olmos Y, Gong C, et al: SIRT6 modulates paclitaxel and epirubicin resistance and survival in breast cancer. Carcinogenesis 34: 1476-1486, 2013.

21. Wang P, Guan YF, Du H, Zhai QW, Su DF and Miao CY: Induction of autophagy contributes to the neuroprotection of nicotinamide phosphoribosyltransferase in cerebral ischemia. Autophagy 8: 77-87, 2012.

22. Filippi BM, Yang CS, Tang C and Lam TK: Insulin activates Erk1/2 signaling in the dorsal vagal complex to inhibit glucose production. Cell Metab 16: 500-510, 2012.

23. Wang P, Xu TY, Guan YF, Su DF, Fan GR and Miao CY: Perivascular adipose tissue-derived visfatin is a vascular smooth muscle cell growth factor: role of nicotinamide mononucleotide. Cardiovasc Res 81: 370-380, 2009.

24. Nezis IP, Shravage BV, Sagona AP, Johansen T, Baehrecke EH and Stenmark H: Autophagy as a trigger for cell death: autophagic degradation of inhibitor of apoptosis dBruce controls DNA fragmentation during late oogenesis in Drosophila. Autophagy 6: $1214-1215,2010$

25. Wang P, Tian WW, Song J, Guan YF and Miao CY: Deficiency of $\mathrm{NG}^{+}$cells contributes to the susceptibility of stroke-prone spontaneously hypertensive rats. CNS Neurosci Ther 17: 327-332, 2011.

26. Wang P, Xu TY, Guan YF, et al: Nicotinamide phosphoribosyltransferase protects against ischemic stroke through SIRT1-dependent adenosine monophosphate-activated kinase pathway. Ann Neurol 69: 360-374, 2011.

27. Wang $\mathrm{P}$, Yang FJ, Du $\mathrm{H}$, et al: Involvement of leptin receptor long isoform (LepRb)-STAT3 signaling pathway in brain fat mass- and obesity-associated (FTO) downregulation during energy restriction. Mol Med 17: 523-532, 2011.
28. Wang P, Zhang RY, Song J, et al: Loss of AMP-activated protein kinase- $\alpha 2$ impairs the insulin-sensitizing effect of calorie restriction in skeletal muscle. Diabetes 61: 1051-1061, 2012.

29. Devarajan A, Grijalva VR, Bourquard N, et al: Macrophage paraoxonase 2 regulates calcium homeostasis and cell survival under endoplasmic reticulum stress conditions and is sufficient to prevent the development of aggravated atherosclerosis in paraoxonase 2 deficiency/apoE-/- mice on a Western diet. Mol Genet Metab 107: 416-427, 2012.

30. Colas E, Perez C, Cabrera S, et al: Molecular markers of endometrial carcinoma detected in uterine aspirates. Int J Cancer 129: 2435-2444, 2011.

31. Lai CC, Lin PM, Lin SF, et al: Altered expression of SIRT gene family in head and neck squamous cell carcinoma. Tumour Biol 34: 1847-1854, 2013.

32. Van Meter M, Mao Z, Gorbunova V and Seluanov A: SIRT6 overexpression induces massive apoptosis in cancer cells but not in normal cells. Cell Cycle 10: 3153-3158, 2011

33. Nishimoto $\mathrm{S}$ and Nishida E: MAPK signalling: ERK5 versus ERK1/2. EMBO Rep 7: 782-786, 2006.

34. Wiesenauer CA, Yip-Schneider MT, Wang Y and Schmidt CM: Multiple anticancer effects of blocking MEK-ERK signaling in hepatocellular carcinoma. J Am Coll Surg 198: 410-421, 2004.

35. Li Y, Xu W, McBurney MW and Longo VD: SirT1 inhibition reduces IGF-I/IRS-2/Ras/ERK1/2 signaling and protects neurons. Cell Metab 8: 38-48, 2008.

36. Zhao Y, Luo P, Guo Q, et al: Interactions between SIRT1 and MAPK/ERK regulate neuronal apoptosis induced by traumatic brain injury in vitro and in vivo. Exp Neurol 237: 489-498, 2012. 